\title{
Cold Gas in the Milky Way's Nuclear Wind
}

Enrico M. Di Teodoro ${ }^{1,2,3}$, N. M. McClure-Griffiths ${ }^{2}$, Felix J. Lockman ${ }^{4}$ \& Lucia Armillotta ${ }^{5,2}$

${ }^{1}$ Department of Physics \& Astronomy, Johns Hopkins University, Baltimore, MD 21218, USA

${ }^{2}$ Research School of Astronomy and Astrophysics - The Australian National University, Canberra, ACT, 2611, Australia

${ }^{3}$ Space Telescope Science Institute, Baltimore, MD 21218, USA

${ }^{4}$ Green Bank Observatory, Green Bank, WV 24944, USA

${ }^{5}$ Department of Astrophysical Sciences, Princeton University, Princeton, NJ 08544

The centre of the Milky Way is the site of several high-energy processes that have strongly impacted the inner regions of our Galaxy. Activity from the super-massive black hole, Sgr A*, and/or stellar feedback from the inner molecular ring ${ }^{1}$ expel matter and energy from the disc in the form of a galactic wind ${ }^{2}$. Multiphase gas has been observed within this outflow, from hot highly-ionized $^{3,4}\left(T \simeq 10^{6} \mathbf{K}\right)$, to warm ionized ${ }^{5,6}\left(T \simeq 10^{4-5} \mathbf{K}\right)$ and cool atomic gas as $^{7,8}$ ( $T \simeq 10^{3-4} \mathbf{K}$ ). To date, however, there has been no evidence of the cold and dense molecular phase ( $T \simeq 10-100 \mathbf{K})$. Here we report the first detection of molecular gas outflowing from the centre of our Galaxy. This cold material is associated with atomic hydrogen clouds travelling in the nuclear wind ${ }^{8}$. The morphology and the kinematics of the molecular gas, resolved on $\sim 1$ pc scale, indicate that these clouds are mixing with the warmer medium and are possibly being disrupted. The data also suggest that the mass of molecular gas driven out is not negligible and could impact the rate of star formation in the central regions. The presence of this cold, dense, high-velocity gas is puzzling, as neither Sgr $\mathrm{A}^{*}$ at its current level of activity, nor star formation in the inner Galaxy seem viable sources for this material.

At a distance of only $8.2 \mathrm{kpc}^{9}$, the Galactic Centre provides a unique laboratory for studying the complex physical processes that occur within a galactic outflow. The Fermi Bubbles ${ }^{2,10}$, two giant lobes extending up to $\sim 10 \mathrm{kpc}$ from the Galactic plane, are thought to outline the current boundaries of the Milky Way's nuclear wind. Several hundred neutral gas clouds have been found recently within this volume through observations of the atomic hydrogen (HI) line at $\lambda=21 \mathrm{~cm}^{7,8}$. 
Figure 1 shows a column density map of HI clouds in the nuclear wind ${ }^{8}$ detected with the Green Bank Telescope (GBT). Although the bulk of the cloud population lies within the boundaries of the Fermi Bubbles (green dashed line ${ }^{11}$ ), it has not been established whether this outflowing HI gas arises from the same event that generated the Fermi Bubbles. These clouds were identified through their anomalous line-of-sight velocities, which are incompatible with Galactic rotation and can instead be described with a bi-conical wind model in which clouds accelerate from the Galactic Centre reaching a maximum velocity of $330 \mathrm{~km} \mathrm{~s}^{-1}$ after about $2.5 \mathrm{kpc}^{8,12}$. To assess whether outflowing HI structures carry molecular gas, we targeted two objects (MW-C1 and MW$\mathrm{C} 2$, hereinafter), highlighted by red boxes in Figure 1 , in the ${ }^{12} \mathrm{CO}(2 \rightarrow 1)$ emission line at 230.538 $\mathrm{GHz}$ with the 12m Atacama Pathfinder Experiment (APEX) telescope. These two clouds have relatively high $\mathrm{HI}$ column densities $\left(>10^{19} \mathrm{~cm}^{-2}\right.$ ) and show an elongated head-to-tail morphology along the direction pointing away from the Galactic Centre. We mapped both clouds in ${ }^{12} \mathrm{CO}(2 \rightarrow 1)$ emission over a $15^{\prime} \times 15^{\prime}$ field centred on the peak of the HI emission, at a spatial resolution of $28^{\prime \prime}$ (Full Width at Half Maximum, FWHM), corresponding to $\sim 1 \mathrm{pc}$ at the distance of the Galactic Centre, and a spectral resolution of $0.25 \mathrm{~km} \mathrm{~s}^{-1}$. These data revealed for the first time molecular gas outflowing from the centre of our Galaxy.

Figure 2 shows HI column-density maps (left, blue colorscale) from GBT observations and integrated brightness temperature maps (right, heat colorscale) from the ${ }^{12} \mathrm{CO}(2 \rightarrow 1)$ line with APEX for MW-C1 (top) and MW-C2 (bottom). Higher resolution HI data from the Australia Telescope Compact Array (ATCA) for MW-C2 are overlaid as contours on the CO map. CO velocity fields and three representative spectra across each field are displayed in Figure 3. CO emission is detected in both HI clouds, with significant morphological and kinematical differences between them. MW-C1 shows five, distinct, compact clumps of molecular gas concentrated towards the part of the HI cloud that faces the Galactic Centre (arrows in Figure 2). At least three clumps have a velocity gradient along the direction pointing towards the tail of the HI cloud. All the CO emission in MW-C1 lies in the velocity range $V_{\mathrm{LSR}} \simeq 160-170 \mathrm{~km} \mathrm{~s}^{-1}$. Typical FWHM line widths are $\sim 2-3 \mathrm{~km} \mathrm{~s}^{-1}$ (see spectra in Figure 3). In contrast, in MW-C2, most of the CO emission is distributed along a filament-like structure, with some fainter and more diffuse clumps in the 
region away from the Galactic Centre. CO emission is spread over a larger velocity range than in MW-C1, spanning $30 \mathrm{~km} \mathrm{~s}^{-1}$ over $V_{\mathrm{LSR}} \simeq 250-280 \mathrm{~km} \mathrm{~s}^{-1}$, and the velocity field does not show any straightforward ordered motion. ${ }^{12} \mathrm{CO}(2 \rightarrow 1)$ line profiles in MW-C2 are much broader than in MW-C1, with FWHM ranging from $\sim 5-12 \mathrm{~km} \mathrm{~s}^{-1}$.

The observed features indicate that cold gas in MW-C2 is interacting and mixing with the surrounding medium more efficiently than in $\mathrm{MW}-\mathrm{C} 1$, resulting in a more turbulent molecular gas. An interpretation of the differences in the morpho-kinematics of the molecular gas in the two clouds is that we are witnessing two evolutionary stages of a cold cloud being disrupted by the interaction with a hot flow. Our idealized bi-conical wind model ${ }^{12}$ with a maximum wind velocity of $330 \mathrm{~km} \mathrm{~s}^{-1}$ places MW-C1 at a distance of $0.8 \mathrm{kpc}$ and MW-C2 at a distance of $1.8 \mathrm{kpc}$ from the Galactic Centre, implying that MW-C2 may have been within the nuclear outflow twice as long (7 Myr vs 3 Myr). Our model also predicts that MW-C2 is moving faster than MW-C1 $\left(\sim 300 \mathrm{~km} \mathrm{~s}^{-1}\right.$ $\mathrm{vs} \sim 240 \mathrm{~km} \mathrm{~s}^{-1}$ ). In the classical picture where cold gas is entrained in the hot wind, MW-C1 may therefore represent an early stage of the interaction with the surrounding medium, where molecular gas is still relatively intact and undisturbed near the initial dense core, while molecular gas in MWC2 could have been stripped off from its core, resulting in a disordered morphology/velocity field and broader linewidths. However, the observed characteristics of the two clouds may also be explained in terms of different local conditions of the hot outflow. A larger and more complete sample of molecular gas detections in outflowing clouds is needed to have a more robust picture.

The two clouds analysed in this work have atomic gas masses of $M_{\text {at }} \simeq 220 \mathrm{M}_{\odot}(\mathrm{MW}-\mathrm{C} 1)$ and $M_{\mathrm{at}} \simeq 800 \mathrm{M}_{\odot}(\mathrm{MW}-\mathrm{C} 2)$, as derived from Hi data. All mass measurements from observations are scaled by a factor 1.36 to account for the presence of Helium. It is not straightforward to estimate the mass of molecular matter, as the gas may have a significant opacity in the ${ }^{12} \mathrm{CO}(2 \rightarrow 1)$ line, and the appropriate $\mathrm{CO}-$ to- $\mathrm{H}_{2}$ conversion factor $X_{\mathrm{CO}}$ in the Milky Way's wind is unknown. We used the observed $\mathrm{CO}$ integrated brightness temperatures, cloud radii and line widths to constrain acceptable $X_{\mathrm{CO}}$ values by means of chemical and thermal modelling of a cloud subject to dissociation by photons and cosmic rays. We found that $X_{\mathrm{CO}}$ for the ${ }^{12} \mathrm{CO}(2 \rightarrow 1)$ transition in our clouds lies in the range $\simeq 2-40 \times 10^{20} \mathrm{~cm}^{-2}\left(\mathrm{~K} \mathrm{~km} \mathrm{~s}^{-1}\right)^{-1}$. The lowest value, 
$X_{\mathrm{CO}}=2 \times 10^{20} \mathrm{~cm}^{-2}\left(\mathrm{~K} \mathrm{~km} \mathrm{~s}^{-1}\right)^{-1}$, is consistent with the Galactic conversion factor ${ }^{13}$, and was used to derive lower limits to the molecular gas mass. We obtained a $M_{\text {mol }} \gtrsim 380 \mathrm{M}_{\odot}$ for MW-C1 and $M_{\text {mol }} \gtrsim 375 \mathrm{M}_{\odot}$ for MW-C2, implying molecular-to-total gas mass fractions $f_{\text {mol }}=M_{\text {mol }} /\left(M_{\text {mol }}+M_{\text {at }}\right) \gtrsim 0.64$ and $\gtrsim 0.32$, respectively. We emphasise that these values are lower bounds and the molecular gas mass may be higher by a factor of ten. As a consequence, the total mass of molecular gas in the nuclear wind of the Milky Way is large. Under the conservative assumption of an average $f_{\mathrm{mol}} \simeq 0.3-0.5$ for all outflowing HI clouds in the GBT sample, and based on the atomic outflow rate ${ }^{8}$ of $\dot{M}_{\text {at }} \sim 0.1 \mathrm{M}_{\odot} \mathrm{yr}^{-1}$, we estimated an outflow rate of $\dot{M}_{\text {mol }} \gtrsim 0.05-0.1 \mathrm{M}_{\odot} \mathrm{yr}^{-1}$ in molecular gas. This value is of the same order of magnitude of the star formation rate (SFR) of the Central Molecular Zone ${ }^{14}$ (CMZ), implying a molecular gas loading factor $\eta=\dot{M}_{\text {mol }} / \mathrm{SFR}$ at least of the order of unity at a distance $\sim 1 \mathrm{kpc}$ from the Galactic plane, a value similar to that estimated in nearby starburst galaxies ${ }^{15}$. This cold outflow affects the gas cycle in the inner Galaxy and may constitute an important mechanism to regulate the star formation activity in the CMZ.

From a theoretical point of view, such a large amount of high-velocity molecular gas is puzzling ${ }^{16}$. It is believed that cool gas in a disc can be lifted and accelerated by both drag force from a hot outflow ${ }^{17}$ and by radiation pressure ${ }^{18}$. This requires a source of strong thermal feedback and/or radiation feedback. The Milky Way does not currently have an active galactic nucleus (AGN), nor is the SFR of the inner Galaxy comparable to that of starburst galaxies with known molecular winds (e.g. NGC253 $3^{15}$ ). Current simulations of AGN-driven winds have focussed on very powerful $\mathrm{AGN}^{19,20}$ and there have been no investigations studying whether a relatively small black hole like Sgr A* could expel large amounts of cold gas, even if it had undergone a period of activity in the recent past. On the other hand, the current SFR of the CMZ is not large enough to explain the estimated outflow rate of cold gas ${ }^{21}$ and no observational evidence to date suggests a significant change in the SFR of the CMZ in the last few $\mathrm{Myr}^{22}$. A scenario where the star formation in the CMZ is episodic on a longer cycle ${ }^{23,24}(10-50 \mathrm{Myr})$ and is currently near a minimum might help to partly reconcile the observed and predicted cool gas mass loading rates, although our wind model suggests that lifetimes of cold clouds are shorter than $10 \mathrm{Myr}$. Cosmic 
rays are also believed to contribute pressure on cold gas $^{25}$, but their role is only just starting to be understood and needs observational constraints. Moreover, in either an AGN-driven or a starburstdriven wind, the extent to which cold gas survives under acceleration is a matter of debate ${ }^{17,26}$, and several different mechanisms have been investigated to extend the lifetime of cool gas in a hot wind (e.g., magnetic fields ${ }^{27}$, thermal conduction ${ }^{28}$ ). An alternative scenario has been recently proposed where high-velocity cool neutral gas $\left(T<10^{4}\right)$ forms directly within the outflow as a consequence of mixing between slow-moving cool clouds and the fast-moving hot wind ${ }^{29,30}$. This mechanism overcomes the problem of accelerating dense material without disrupting it and may explain the high velocities observed in cool outflows. However, current simulations are not able to trace the gas down to the molecular phase.

In conclusion, our first detection of ouflowing cold molecular gas in the Milky Way is a challenge for current theories of galactic winds in regular star-forming galaxies, as none of the above processes seems able to easily explain the presence of fast molecular gas in the Milky Way's wind. Targeted observations of molecular gas tracers in the Milky Way's nuclear wind promise to contribute significantly to our understanding of these fascinating phenomena. 


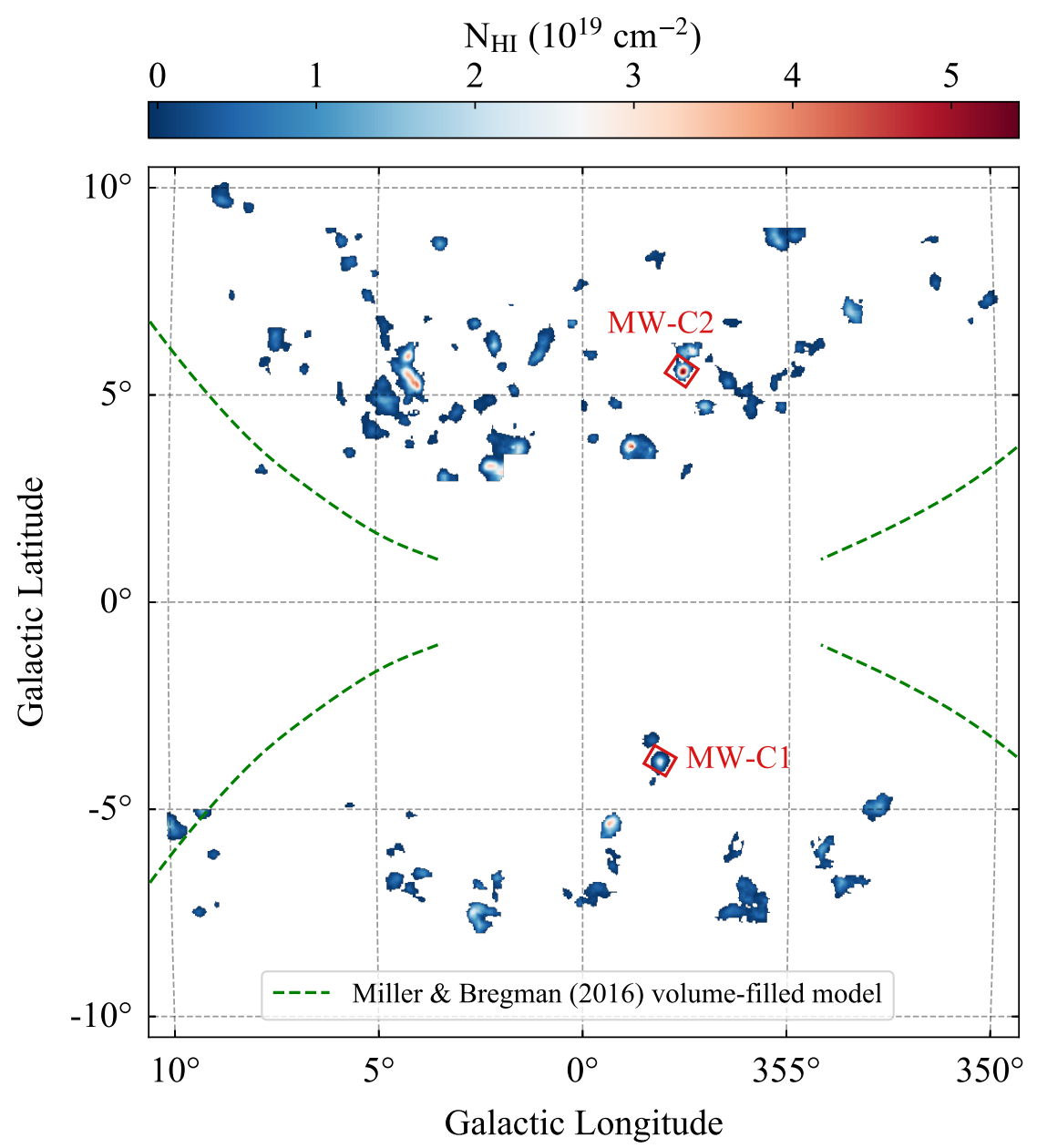

Figure 1: Atomic hydrogen gas outflowing from the Galactic Centre. Blue-red colorscale is the colum density of anomalous HI clouds in the MW nuclear wind detected with the $\mathrm{GBT}^{8}$. The green-dashed line is the boundary of a volume-filled model for the Fermi Bubbles ${ }^{11}$. The two HI clouds observed in the ${ }^{12} \mathrm{CO}(2 \rightarrow 1)$ line with APEX are enclosed by red boxes. 

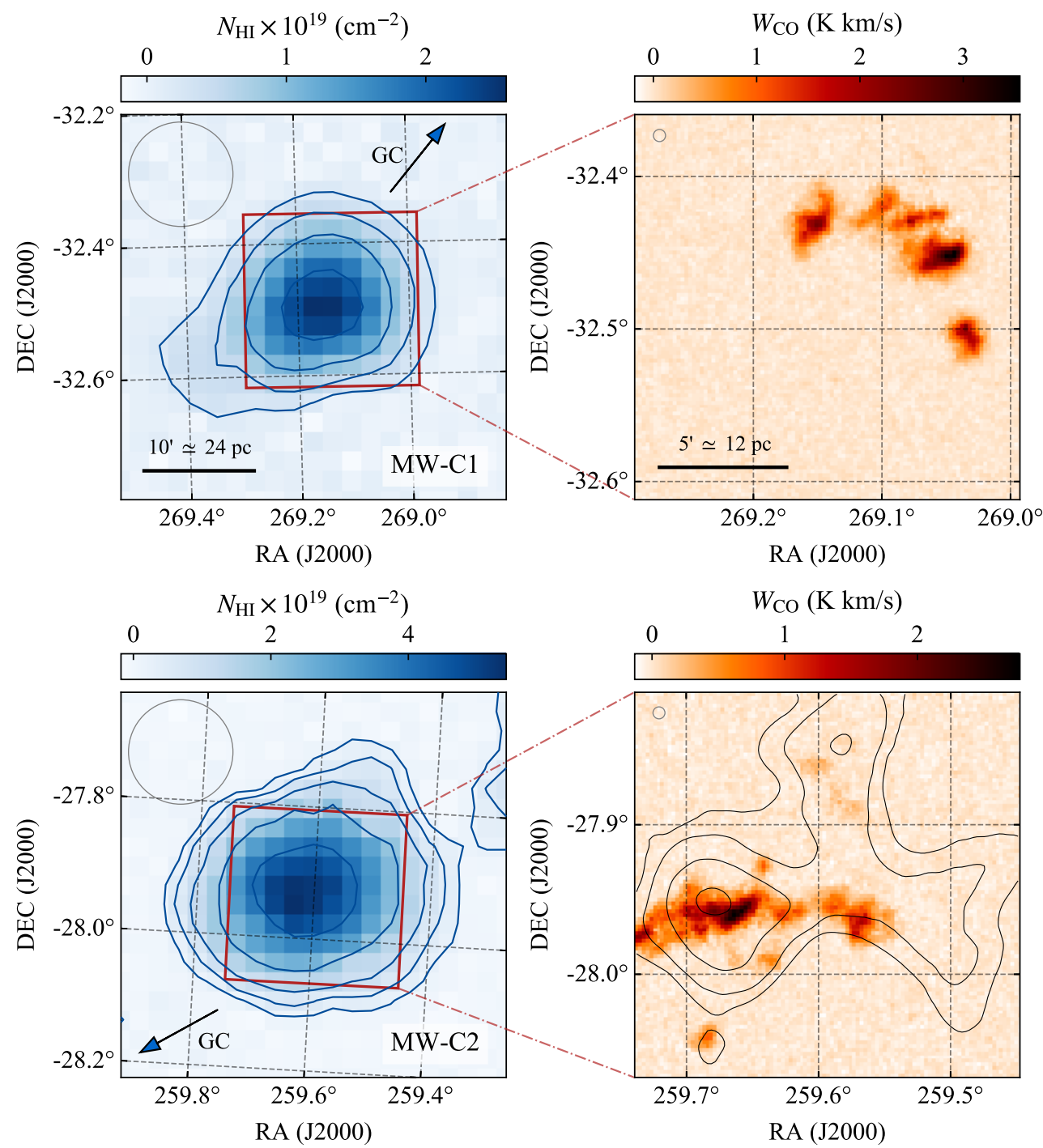

Figure 2: Atomic hydrogen and molecular gas in two clouds, MW-C1 and MW-C2 in the Milky Way nuclear wind. Top panels show MW-C1, bottom panels MW-C2. On the left, HI column density maps from GBT data ${ }^{8}$ at an angular resolution of $570^{\prime \prime}$. Black arrows point toward the Galactic Centre. Red boxes highlight the $15^{\prime} \times 15^{\prime}$ fields observed with APEX. Contour levels are at $(0.2,0.5,1,2,4) \times 10^{19} \mathrm{~cm}^{-2}$. On the right, integrated ${ }^{12} \mathrm{CO}(2 \rightarrow 1)$ brightness temperature maps from APEX data at $28^{\prime \prime}$ resolution. Hi contours at $(4,8,16,24) \times 10^{20} \mathrm{~cm}^{-2}$ from ATCA data at $137^{\prime \prime}$ resolution are overlaid on the MW-C2 map. Circles at the top-left of each panel show the angular resolution of the telescopes. 

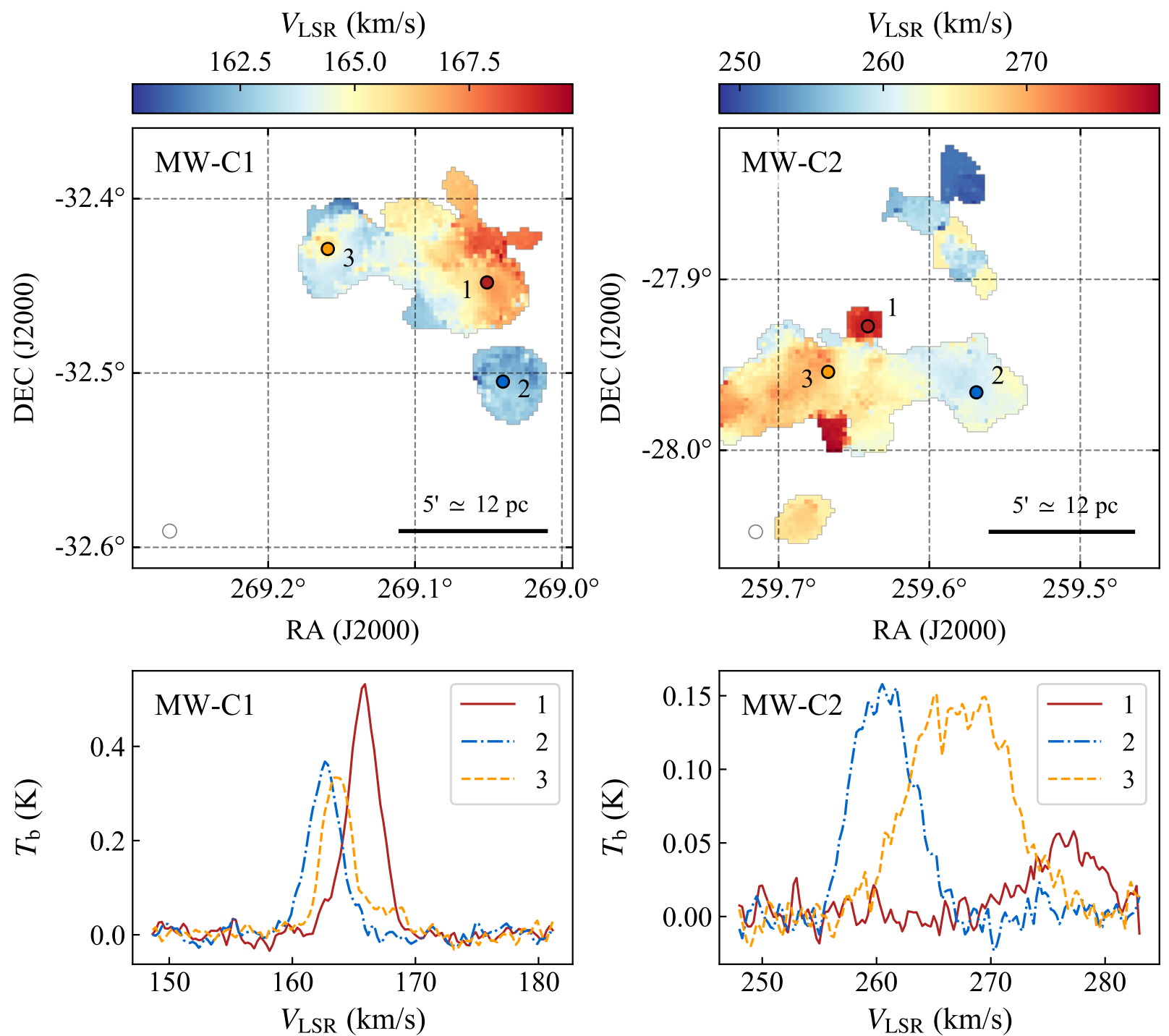

Figure 3: Molecular gas kinematics in MW-C1 and MW-C2Left panels show MW-C1, right panels MW-C2. Velocity fields derived through a Gaussian fit to the ${ }^{12} \mathrm{CO}(2 \rightarrow 1)$ data are displayed in the top panels. Bottom panels show ${ }^{12} \mathrm{CO}(2 \rightarrow 1)$ spectra at the positions labelled on the top panels. Note the difference in velocity spread and line shape between MW-C1 and MW-C2. 


\section{Methods}

Observations and data reduction. Observations of the ${ }^{12} \mathrm{CO}(2 \rightarrow 1)$ emission line at $230.538 \mathrm{GHz}$ were made with the $12 \mathrm{~m} \mathrm{APEX}$ antenna ${ }^{31}$ using the PI230 heterodyne receiver (ESO project ID 0104.B-0106A; P.I. Di Teodoro E. M.). The spectrometer ${ }^{32}$ covers a bandwidth of $8 \mathrm{GHz}$ at a spectral resolution of $61 \mathrm{kHz}$, corresponding to a velocity resolution of about $0.08 \mathrm{~km} \mathrm{~s}^{-1}$ at 230 GHz. The beam size at this frequency is 27.8 " (FWHM), the main-beam efficiency is 0.72 and the $\mathrm{Jy} / \mathrm{K}$ conversion factor is $40 \pm 3$. We observed our targets in on-the-fly position-switching mode, integrating for 1 second every $9^{\prime \prime}$. Both fields were $15^{\prime} \times 15^{\prime}$ wide, centred at $(\alpha, \delta)_{\mathrm{J} 2000}=$ $\left(17 \mathrm{~h} 56 \mathrm{~m} 34.0 \mathrm{~s},-32^{\circ} 29^{\prime} 14^{\prime \prime}\right)$ for MW-C1 and at $(\alpha, \delta)_{\mathrm{J} 2000}=\left(17 \mathrm{~h} 18 \mathrm{~m} 22.2 \mathrm{~s},-27^{\circ} 56^{\prime} 28^{\prime \prime}\right)$ for MW-C2. Observed regions are shown as red boxes in Figure 1 and in Figure 2. Total integration time was approximately $25 \mathrm{~h}$ for each field. Throughout the observing session (SeptemberNovember 2019), the precipitable water vapour (PWV) varied between 0.6 and $3 \mathrm{~mm}$.

We reduced the data using the Continuum and Line Analysis Single-dish Software (CLASS) from the GILDAS package ${ }^{33}$. A first-order baseline was subtracted from calibrated spectra by interpolating the channels outside the velocity windows where we expected to see the emission based on the Hi observations. Spectra were then smoothed in velocity and mapped onto a grid with a pixel size of $9^{\prime \prime}$ and a channel width of $0.25 \mathrm{~km} \mathrm{~s}^{-1}$. The root mean square noise $\left(\sigma_{\text {rms }}\right)$ in the final datacubes is $65 \mathrm{mK}$ and $55 \mathrm{mK}$ in a $0.25 \mathrm{~km} \mathrm{~s}^{-1}$ channel for MW-C1 and MW-C2, respectively.

Atomic gas and molecular gas mass. The HI GBT data ${ }^{8}$ and the ${ }^{12} \mathrm{CO}(2 \rightarrow 1)$ APEX data were analyzed to estimate atomic and molecular gas masses, respectively. First, the 3D source finder DUCHAMP ${ }^{34}$ was applied to the data cubes to identify regions of significant emission. During this process, we set a primary threshold to identify emission peaks at $5 \times \sigma_{\text {rms }}$ and reconstructed sources by adding pixels down to a secondary threshold of $2.5 \times \sigma_{\text {rms }}$, where $\sigma_{\text {rms }}$ is the rms noise in a given datacube.

The column density at a given position $(x, y)$ on the sky can be written as: 


$$
N_{\mathrm{H}}(x, y)=C \int T_{\mathrm{b}}(x, y, v) d v
$$

where the integral considers only pixels in a detection, $T_{\mathrm{b}}$ is the line brightness temperature, $d v$ is the channel width $\left(1 \mathrm{~km} \mathrm{~s}^{-1}\right.$ for GBT and $0.25 \mathrm{~km} \mathrm{~s}^{-1}$ for APEX) and $C$ is a constant. For the HI line, under the assumption that the gas is optically thin, the constant is ${ }^{35} C=1.82 \times$ $10^{18} \mathrm{~cm}^{-2}\left(\mathrm{~K} \mathrm{~km} \mathrm{~s}^{-1}\right)^{-1}$. For CO lines, this constant is also known as the CO-to- $\mathrm{H}_{2}$ conversion factor $X_{\mathrm{CO}}{ }^{13}$. Because the conversion factor in the nuclear wind can not be constrained with current data, we used the value estimated in molecular clouds in the MW's disc ${ }^{36}, X_{\mathrm{CO}}=$ $2 \times 10^{20} \mathrm{~cm}^{-2}\left(\mathrm{~K} \mathrm{~km} \mathrm{~s}^{-1}\right)^{-1}$. We checked this $X_{\mathrm{CO}}$ value against the predictions from radiative transfer models, described in the next Section, and found that the Galactic value is probably a lower limit for clouds in the nuclear wind.

The total mass of gas can finally be calculated as:

$$
M=1.36 m D^{2} \int N_{\mathrm{H}}(x, y) d x d y
$$

where the factor 1.36 takes into account Helium, $D \simeq 8.2 \mathrm{kpc}$ is the adopted distance to the clouds, $m$ is the mass of atomic/molecular hydrogen for atomic/molecular gas, $d x$ and $d y$ are the pixel sizes in radians (105" for GBT, $9^{\prime \prime}$ for APEX). Observed properties and estimated masses are summarized in Extended Data Table 1.

Radiative transfer models. We used the chemistry and radiative transfer code DESPOTIC ${ }^{37}$ to constrain the CO-to- $\mathrm{H}_{2}$ conversion factor of the clouds. DESPOTIC computes the chemical and thermal state of an optically-thick cloud given its volume density and column density. The gas turbulent velocity dispersion was assumed to be $1-5 \mathrm{~km} \mathrm{~s}^{-1}$ (see Figure 3 ) in our modelling. The chemical equilibrium calculation uses solar abundances for dust and all elements in the $\mathrm{H}-\mathrm{C}-\mathrm{O}$ chemical network $^{38}$, while the thermal equilibrium calculation includes heating by cosmic rays, grain photoelectric effect, cooling by the lines of HI, CII, CI, OI, and CO, and collisional energy 
exchange between dust and gas. Level populations were calculated using an escape probability method, with escape probabilities estimated using DESPOTIC's spherical geometry option.

We investigated different combinations of interstellar radiation field $\chi$ and cosmic ray ionization rate $\zeta$ through a set of DESPOTIC models with $\log \left(\chi / G_{0}\right)=[-1,0,1,2]$, where $G_{0}$ is the Solar radiation field ${ }^{39}$ and $\log \left(\zeta / \mathrm{s}^{-1}\right)=[-16,-15,-14]$. The interstellar radiation field was varied between sub-Solar $\left(\chi \simeq 0.1 G_{0}\right)$ and highly super-Solar $\left(\chi \simeq 100 G_{0}\right.$, a value representative of a highly star-forming environment as the CMZ). The cosmic ray ionization rate ranges from the value measured in the Solar neighbourhood ${ }^{40}\left(\zeta \simeq 10^{-16} \mathrm{~s}^{-1}\right)$ to the estimated upper limit for the $\mathrm{CMZ}^{41}\left(\zeta \simeq 10^{-14} \mathrm{~s}^{-1}\right)$. We stress that our $\mathrm{CO}$ clouds lie at about $1 \mathrm{kpc}$ from the Galactic Plane and that both the interstellar radiation field and the cosmic ray ionization rate are expected to drop with distance from the disc. Therefore, although the estimated values of $\chi$ and $\zeta$ in the $\mathrm{CMZ}$ are orders of magnitude higher than in the Solar neighbourhood, models with intermediate interstellar radiation field and cosmic ray ionization rate should be more representative of conditions high in the Milky Way's wind.

For each model, DESPOTIC returned the ${ }^{12} \mathrm{CO}(2 \rightarrow 1)$ integrated brightness temperatures $\left(W_{\mathrm{CO}}\right)$ as a function of number density $\left(n_{\mathrm{H}_{2}}\right)$ and column density $\left(N_{\mathrm{H}_{2}}\right)$ of molecular Hydrogen. We only considered solutions consistent with the observed integrated brightness temperature $\left(1-5 \mathrm{~K} \mathrm{kms}^{-1}\right.$, see Figure 2) and observed cloud radius $R=0.75 n_{\mathrm{H}_{2}} / N_{\mathrm{H}_{2}}(1-5 \mathrm{pc})$ and we calculated the expected CO-to- $\mathrm{H}_{2}$ conversion factor $X_{\mathrm{CO}}=N_{\mathrm{H}_{2}} / W_{\mathrm{CO}}$ for the ${ }^{12} \mathrm{CO}(2 \rightarrow 1)$ transition. We found that there are no acceptable solutions for a strong interstellar radiation field $\left(\log \left(\chi / G_{0}\right) \geq 1\right)$, indicating that molecular clouds with the observed properties can not exist in the presence of a CMZ-like radiation field. Instead, models with Solar and sub-Solar radiation fields returned solutions compatible with the observational constraints for any cosmic ray ionization field. An interstellar radiation field weaker than the one produced in the CMZ is therefore more representative of the environment at $1 \mathrm{kpc}$ above the Galactic Centre. Predicted $X_{\mathrm{CO}}$ varies by an order of magnitude, ranging between $\simeq 2 \times 10^{20} \mathrm{~cm}^{-2}\left(\mathrm{~K} \mathrm{~km} \mathrm{~s}^{-1}\right)^{-1}$ and $\simeq 4 \times 10^{21} \mathrm{~cm}^{-2}\left(\mathrm{~K} \mathrm{~km} \mathrm{~s}^{-1}\right)^{-1}$, depending on the combination of radiation field and cosmic ray ionization rate. The value of 
$X_{\mathrm{CO}}=2 \times 10^{20} \mathrm{~cm}^{-2}\left(\mathrm{~K} \mathrm{~km} \mathrm{~s}^{-1}\right)^{-1}$ commonly assumed in the Milky Way disc ${ }^{13}$ and used in this paper is consistent with the smallest values returned by our radiative transfer models, obtained with a weak, sub-Solar, radiation field and a Solar-like cosmic ray ionization rate of $\zeta=10^{-16} \mathrm{~s}^{-1}$. As a consequence, the molecular gas masses calculated in this work likely represent lower limits to the real cold gas mass in our $\mathrm{CO}$ clouds.

Wind kinematic model. To estimate the position, velocity and lifetime of MW-C1 and MW-C2, we used a bi-conical wind model ${ }^{8,12}$ calibrated on the full population of HI clouds. This model is based on the assumption that clouds were launched from a small region close to the centre of the Galaxy and are moving with a purely radial velocity $V_{\mathrm{w}}(r)$, where $r$ is the distance from the Galactic Centre. For simplicity, we considered models of the form:

$$
V_{\mathrm{w}}(r)= \begin{cases}V_{\mathrm{i}}+\left(V_{\max }-V_{\mathrm{i}}\right) \frac{r}{r_{\mathrm{s}}} & \text { for } r<r_{\mathrm{s}} \\ V_{\max } & \text { for } r \geq r_{\mathrm{s}}\end{cases}
$$

where $V_{\mathrm{i}}$ is the initial velocity at $r=0$ and $r_{\mathrm{s}}$ is the scale distance at which the maximum velocity $V_{\max }$ is reached. Eq. 3 describes a kinematic model where clouds are subject to a constant acceleration up to $r_{\mathrm{s}}$ and maintain a constant velocity at distances $r \geq r_{\mathrm{s}}$. Although Eq. 3 is purely empirical and chosen to reproduce the HI data, recent hydrodynamical simulations of starburstdriven winds have found qualitatively similar trends for the cool gas velocity with distance ${ }^{30}$. The local standard of rest (LSR) velocity $V_{\mathrm{LSR}}$ of a cloud travelling in the wind and seen at Galactic coordinates $(\ell, b)$ can be written as:

$$
V_{\mathrm{LSR}}(\ell, b, r)=V_{\mathrm{w}}(r)(\sin \phi \sin b-\cos \phi \cos b \cos (\ell+\theta)]-V_{0} \sin \ell \sin b
$$

where the polar angle $\phi$ and the azimuthal angle $\theta$ can easily be written as a function of $(\ell, b, r)^{8}$ and $V_{0}=240 \mathrm{~km} \mathrm{~s}^{-1}$ is the rotation velocity of the LSR around the Galactic Centre ${ }^{42}$. In our model, clouds are restricted inside a bi-cone with half opening angle $\phi_{\max }$. We constrained the four free parameters of this model, i.e. $V_{\mathrm{i}}, V_{\max }, r_{\mathrm{s}}$ and $\phi_{\max }$, by matching the LSR velocity distributions 
Extended Data Table 1: Measured and derived properties of molecular gas clouds outflowing from the Milky Way. We list: Galactic coordinates $(\ell, b)$; height from the Galactic plane $(z)$; distance from the Galactic Centre $(r)$ from our bi-conical outflow model ${ }^{12}$; peak ${ }^{12} \mathrm{CO}(2 \rightarrow 1)$ brightness temperature $\left(T_{\mathrm{b}, \text { peak }}\right)$; typical CO line widths (FWHM); velocity range of CO line in local standard of rest (LSR); lower limits to molecular masses $\left(M_{\text {mol }}\right)$, derived from ${ }^{12} \mathrm{CO}(2 \rightarrow 1)$ data; atomic gas masses $\left(M_{\text {at }}\right)$, derived from Hi data. Masses include Helium.

\begin{tabular}{cccccccccc}
\hline \hline & $\begin{array}{c}\ell \\
\left(^{\circ}\right)\end{array}$ & $\begin{array}{c}b \\
\left({ }^{\circ}\right)\end{array}$ & $\begin{array}{c}z \\
(\mathrm{kpc})\end{array}$ & $\begin{array}{c}T_{\mathrm{b}, \text { peak }} \\
(\mathrm{kpc})\end{array}$ & $\begin{array}{c}\text { FWHM } \\
(\mathrm{K})\end{array}$ & $\begin{array}{c}\text { Vel. range } \\
\left(\mathrm{km} \mathrm{s}^{-1}\right)\end{array}$ & $\begin{array}{c}M_{\text {mol }} \\
\left(\mathrm{km} \mathrm{s}^{-1}\right)\end{array}$ & $\begin{array}{c}M_{\mathrm{at}} \\
\left(\mathrm{M}_{\odot}\right)\end{array}$ & $\left(\mathrm{M}_{\odot}\right)$ \\
\hline \hline MW-C1 & 358.14 & -3.84 & 0.6 & 0.8 & 1.5 & $2-3$ & $160-170$ & 380 & 220 \\
MW-C2 & 357.58 & 5.56 & 0.9 & 1.8 & 0.5 & $5-12$ & $250-280$ & 375 & 800 \\
\hline
\end{tabular}

predicted by our model with that observed from the HI cloud population ${ }^{8,12}$. Our fiducial model is a bi-conical wind with opening angle $\phi_{\max }=70^{\circ}$, where clouds accelerate from an initial velocity $V_{\mathrm{i}}=200 \mathrm{~km} \mathrm{~s}^{-1}$ to a maximum velocity $V_{\max }=330 \mathrm{~km} \mathrm{~s}^{-1}$ at $r_{\mathrm{s}}=2.5 \mathrm{kpc}^{12}$. According to this wind model, MW-C1 and MW-C2 have travelled a distance of $0.8 \mathrm{kpc}$ and $1.8 \mathrm{kpc}$ from the Galactic Centre in about $3 \mathrm{Myr}$ and $7 \mathrm{Myr}$, and their current outflow velocity is about $240 \mathrm{~km} \mathrm{~s}^{-1}$ and $300 \mathrm{~km} \mathrm{~s}^{-1}$, respectively.

Acknowledgements E.D.T. and L.A. thank E. Ostriker, C.-G. Kim and J.-G. Kim for useful discussions, and M. Krumholz for support with the DESPOTIC code. E.D.T. was supported by the National Science Foundation under grant 1616177. E.D.T. and N.M.-G. acknowledge the support of the Australian Research Council (ARC) through grant DP160100723. N.M.-G. acknowledges funding from the ARC via Future Fellowship FT150100024. CO observations were made with APEX under ESO proposal 0104.B-0106A. APEX is a collaboration between the Max-Planck-Institut fur Radioastronomie, the European Southern Observatory, and the Onsala Space Observatory. The Green Bank Observatory is a facility of the National Science Foundation operated under a cooperative agreement by Associated Universities, Inc. The Australia Telescope Compact Array is part of the Australia Telescope National Facility which is funded by the Australian Government for operation as a National Facility managed by CSIRO. 
Author contribution E.D.T., N.Mc.-G. and F.J.L. developed the idea for the project. E.D.T. reduced and analysed the APEX data, L.A. ran the radiative transfer models. E.D.T. wrote the paper with direct contributions from N.Mc.-G., F.J.L. and L.A. All authors reviewed the manuscript.

Data availability The APEX raw datasets analysed during the current study will be available at the end of the proprietary period (September 2020) on the ESO archive: http://archive.eso.org/eso/eso_archive_main.html. The GBT raw datasets are publicly available at NRAO archive: https://science.nrao.edu/facilities/gbt/softwareand-tools. The corresponding author will also provide fully-reduced data on reasonable request.

Code availability Software used in this work is publicly available. The GILDAS/CLASS packages for sub-mm data reduction can be found at https://www.iram.fr/IRAMFR/GILDAS. DUCHAMP source finder can be downloaded from https://www.atnf.csiro.au/people/Matthew.Whiting/Duchamp. DESPOTIC radiative transfer code is available at https://bitbucket.org/krumholz/despotic.

Competing Interests The authors declare that they have no competing financial interests.

Correspondence Correspondence and requests for materials should be addressed to Enrico Di Teodoro

\section{References}

1. Molinari, S. et al. A 100 pc Elliptical and Twisted Ring of Cold and Dense Molecular Clouds Revealed by Herschel Around the Galactic Center. ApJ 735, L33 (2011). 1105.5486.

2. Bland-Hawthorn, J. \& Cohen, M. The Large-Scale Bipolar Wind in the Galactic Center. Astrophys. J. 582, 246-256 (2003). astro-ph/ 0208553.

3. Kataoka, J. et al. Suzaku Observations of the Diffuse X-Ray Emission across the Fermi Bubbles’ Edges. Astrophys. J. 779, 57 (2013). 1310.3553.

4. Ponti, G. et al. An X-ray chimney extending hundreds of parsecs above and below the Galactic Centre. Nature 567, 347-350 (2019). 1904.05969.

5. Fox, A. J. et al. Probing the Fermi Bubbles in Ultraviolet Absorption: A Spectroscopic Signature of the Milky Way’s Biconical Nuclear Outflow. ApJ 799, L7 (2015). 1412 . 1480. 
6. Bordoloi, R. et al. Mapping the Nuclear Outflow of the Milky Way: Studying the Kinematics and Spatial Extent of the Northern Fermi Bubble. Astrophys. J. 834, 191 (2017). 1612 . 01578.

7. McClure-Griffiths, N. M. et al. Atomic Hydrogen in a Galactic Center Outflow. ApJ 770, L4 (2013). 1304.7538.

8. Di Teodoro, E. M. et al. Blowing in the Milky Way Wind: Neutral Hydrogen Clouds Tracing the Galactic Nuclear Outflow. Astrophys. J. 855, 33 (2018). 1802 . 02152.

9. Gravity Collaboration et al. Detection of the Schwarzschild precession in the orbit of the star S2 near the Galactic centre massive black hole. Astron. Astrophys 636, L5 (2020). 2004 . 07187.

10. Su, M., Slatyer, T. R. \& Finkbeiner, D. P. Giant Gamma-ray Bubbles from Fermi-LAT: Active Galactic Nucleus Activity or Bipolar Galactic Wind? Astrophys. J. 724, 1044-1082 (2010). 1005.5480 .

11. Miller, M. J. \& Bregman, J. N. The Interaction of the Fermi Bubbles with the Milky Way's Hot Gas Halo. Astrophys. J. 829, 9 (2016). 1607.04906.

12. Lockman, F. J., Di Teodoro, E. M. \& McClure-Griffiths, N. M. Observation of Acceleration of H I Clouds within the Fermi Bubbles. Astrophys. J. 888, 51 (2020). 1911.06864.

13. Bolatto, A. D., Wolfire, M. \& Leroy, A. K. The CO-to- $\mathrm{H}_{2}$ Conversion Factor. ARA\&A 51, 207-268 (2013). 1301.3498.

14. Longmore, S. N. et al. Variations in the Galactic star formation rate and density thresholds for star formation. Mon. Not. R. Astron. Soc. 429, 987-1000 (2013). 1208 . 4256.

15. Bolatto, A. D. et al. Suppression of star formation in the galaxy NGC 253 by a starburst-driven molecular wind. Nature 499, 450-453 (2013). 1307 . 6259.

16. Veilleux, S., Maiolino, R., Bolatto, A. D. \& Aalto, S. Cool Outflows in Galaxies and their Implications. arXiv e-prints arXiv:2002.07765 (2020). 2002.07765. 
17. Scannapieco, E. \& Brüggen, M. The Launching of Cold Clouds by Galaxy Outflows. I. Hydrodynamic Interactions with Radiative Cooling. Astrophys. J. 805, 158 (2015). 1503.06800.

18. Thompson, T. A., Fabian, A. C., Quataert, E. \& Murray, N. Dynamics of dusty radiationpressure-driven shells and clouds: fast outflows from galaxies, star clusters, massive stars, and AGN. Mon. Not. R. Astron. Soc. 449, 147-161 (2015). 1406.5206.

19. Mukherjee, D., Bicknell, G. V., Sutherland, R. \& Wagner, A. Relativistic jet feedback in high-redshift galaxies - I. Dynamics. Mon. Not. R. Astron. Soc. 461, 967-983 (2016). 1606. 01143.

20. Richings, A. J. \& Faucher-Giguère, C.-A. Radiative cooling of swept-up gas in AGN-driven galactic winds and its implications for molecular outflows. Mon. Not. R. Astron. Soc. 478, 3100-3119 (2018). 1710.09433.

21. Armillotta, L., Krumholz, M. R., Di Teodoro, E. M. \& McClure-Griffiths, N. M. The life cycle of the Central Molecular Zone - I. Inflow, star formation, and winds. Mon. Not. R. Astron. Soc. 490, 4401-4418 (2019). 1905.01309.

22. Barnes, A. T. et al. Star formation rates and efficiencies in the Galactic Centre. Mon. Not. $R$. Astron. Soc. 469, 2263-2285 (2017). 1704 . 03572.

23. Krumholz, M. R., Kruijssen, J. M. D. \& Crocker, R. M. A dynamical model for gas flows, star formation and nuclear winds in galactic centres. Mon. Not. R. Astron. Soc. 466, 1213-1233 (2017). 1605.02850 .

24. Armillotta, L., Krumholz, M. R. \& Di Teodoro, E. M. The life cycle of the Central Molecular Zone - II. Distribution of atomic and molecular gas tracers. Mon. Not. R. Astron. Soc. 493, 5273-5289 (2020). 2002.06218.

25. Girichidis, P., Naab, T., Hanasz, M. \& Walch, S. Cooler and smoother - the impact of cosmic rays on the phase structure of galactic outflows. Mon. Not. R. Astron. Soc. 479, 3042-3067 (2018). 1805.09333. 
26. Zhang, D., Thompson, T. A., Quataert, E. \& Murray, N. Entrainment in trouble: cool cloud acceleration and destruction in hot supernova-driven galactic winds. Mon. Not. R. Astron. Soc. 468, 4801-4814 (2017). 1507.01951.

27. McCourt, M., O’Leary, R. M., Madigan, A.-M. \& Quataert, E. Magnetized gas clouds can survive acceleration by a hot wind. Mon. Not. R. Astron. Soc. 449, 2-7 (2015). 1409 . 6719.

28. Armillotta, L., Fraternali, F., Werk, J. K., Prochaska, J. X. \& Marinacci, F. The survival of gas clouds in the circumgalactic medium of Milky Way-like galaxies. Mon. Not. R. Astron. Soc. 470, 114-125 (2017). 1608.05416.

29. Gronke, M. \& Oh, S. P. The growth and entrainment of cold gas in a hot wind. Mon. Not. R. Astron. Soc. 480, L111-L115 (2018). 1806.02728.

30. Schneider, E. E., Ostriker, E. C., Robertson, B. E. \& Thompson, T. A. The Physical Nature of Starburst-driven Galactic Outflows. Astrophys. J. 895, 43 (2020).

31. Güsten, R. et al. The Atacama Pathfinder EXperiment (APEX) - a new submillimeter facility for southern skies -. Astron. Astrophys 454, L13-L16 (2006).

32. Klein, B. et al. High-resolution wide-band fast Fourier transform spectrometers. Astron. Astrophys 542, L3 (2012). 1203.3972.

33. Gildas Team. GILDAS: Grenoble Image and Line Data Analysis Software. Astrophysics Source Code Library (2013). 1305.010.

34. Whiting, M. T. DUCHAMP: a 3D source finder for spectral-line data. Mon. Not. R. Astron. Soc. 421, 3242-3256 (2012). 1201 . 2710.

35. Roberts, M. S. Radio Observations of Neutral Hydrogen in Galaxies, 309 (the University of Chicago Press, 1975).

36. Heyer, M., Krawczyk, C., Duval, J. \& Jackson, J. M. Re-Examining Larson's Scaling Relationships in Galactic Molecular Clouds. Astrophys. J. 699, 1092-1103 (2009). 0809.1397. 
37. Krumholz, M. R. DESPOTIC - a new software library to Derive the Energetics and SPectra of Optically Thick Interstellar Clouds. Mon. Not. R. Astron. Soc. 437, 1662-1680 (2014). 1304.2404 .

38. Gong, M., Ostriker, E. C. \& Wolfire, M. G. A Simple and Accurate Network for Hydrogen and Carbon Chemistry in the Interstellar Medium. Astrophys. J. 843, 38 (2017). 1610.09023.

39. Draine, B. T. Photoelectric heating of interstellar gas. ApJS 36, 595-619 (1978).

40. Indriolo, N. \& McCall, B. J. Investigating the Cosmic-Ray Ionization Rate in the Galactic Diffuse Interstellar Medium through Observations of $\mathrm{H}^{+}{ }_{3}$. Astrophys. J. 745, 91 (2012). 1111.6936.

41. Oka, T. et al. The Central $300 \mathrm{pc}$ of the Galaxy Probed by Infrared Spectra of $\mathrm{H}_{3}^{+}$and CO. I. Predominance of Warm and Diffuse Gas and High $\mathrm{H}_{2}$ Ionization Rate. Astrophys. J. 883, 54 (2019). 1910.04762.

42. Bland-Hawthorn, J. \& Gerhard, O. The Galaxy in Context: Structural, Kinematic, and Integrated Properties. ARA\&A 54, 529-596 (2016). 1602 . 07702. 